\title{
TAILORING RADIO-WAVES, LIGHT AND SOUND WITH METAMATERIALS
}

\author{
Andrea Alù
}

The University of Texas at Austin, Austin, Texas, USA

\begin{abstract}
Metamaterials are artificial materials with unusual bulk properties, based on suitably designed arrays of subwavelength inclusions. They have properties not available in their constituent elements, nor in natural materials at the frequency of interest. These unusual responses are not associated with mixing rules, but they emerge from strong wave-matter interactions, based on carefully engineered meta-atoms and their relative arrangements. In the past decade, metamaterials have opened several exciting directions for basic science, first started in electromagnetics and optics, then expanded into other fields, including acoustics and mechanics. In this paper, I briefly review some recent applications of metamaterials and their potential impact on different engineering frontiers, overcoming long-standing challenges for technology.
\end{abstract}

\section{INTRODUCTION}

The field of metamaterials has seen a continuous and steady growth in the past fifteen years. After initial pioneering work aimed at demonstrating the possibility of negative index of refraction at microwave frequencies [1], resulting from a composite medium with negative real parts of its effective permittivity and permeability, also known as double-negative (DNG) material, the interest has rapidly expanded to the unprecedented electromagnetic properties of these materials, and their potential applications in different engineering fields. These have included the possibility of building ultrathin lenses with subdiffractive resolution properties [2], ultrathin cavity resonators [3] and waveguides [4], and scatterers with unusually large [5] or small [6] cross-section. With the realization of these unusual electromagnetic responses, the interest has rapidly shifted to other fascinating applications, including the possibility of cloaking objects from the incoming radiation [7]-[8], and advancing antenna technology [9]. While the first demonstrations of these unusual concepts were limited to microwave frequencies, the interest in metamaterials expanded in following years to optics, acoustics, and more recently even to other types of waves, including seismic and matter waves.

Among the unusual electromagnetic properties available with metamaterials, also "single-negative (SNG)" materials are popular. These are media for which only one of the material parameters, not both, has a negative real value. This implies that the metamaterial can be properly homogenized and described as a bulk material, which often times requires careful homogenization procedures [10]. SNG media exhibit interesting properties when they are paired in a conjugate manner. These media include epsilonnegative (ENG) media, in which the real part of permittivity is negative but the real part of permeability is positive, and munegative (MNG) media, in which the real part of permeability is negative but the real part of permittivity is positive. When paired together unusual resonances and tunneling arises [11]. The idea of constructing an effective DNG medium by having layers of SNG media has been originally explored by Fredkin and Ron in [12]. Since then, several exciting applications of SNG and DNG materials have been explored in different contexts [13].

In the following, we review a few applications of interest that my group has explored in recent years. I will describe in more detail the impact of these concepts for basic science and engineering applications during my presentation at the conference.

\section{CLOAKING AND RADIO-TRANSPARENT ANTENNAS}

Invisibility has drawn the imagination of mankind for several centuries. With the recent developments in metamaterial technology, the possibility of cloaking objects to incoming electromagnetic radiation has been getting close to a technological reality [6]-[8]. While the cloaking concepts keep inspiring scientists and laymen for their fascinating potential, actually cloaked objects can be of importance also in several practical fields of engineering, including radar-camouflaging, radio-transparent devices, and non-invasive biomedical sensing [14]. At the same time, it should be realized that it is inherently challenging to significantly suppress the scattering of objects many wavelengths large with a passive coating [15], as there are fundamental constraints dictated by causality that limit the overall scattering reduction achievable over a certain bandwidth, as a function of the electrical size of the object. In this regard, one of the most viable and realistic application of cloaking technology focuses in the area of radio-frequency cloaked antennas or radiators, whose size is comparable to the wavelength, and therefore it allows significant scattering suppression over large bandwidths. Our group has pioneered this area, proposing several radio-wave antenna cloaking applications [16]-[18], including the possibility of largely suppressing the electromagnetic signature of conventional antennas using suitably designed metasurfaces, while preserving the possibility of transmitting and receiving signals over large bandwidths. Figure 1, as an example, shows a cloaked dipole antenna for cellular communications, highlighting significantly suppressed radar cross section, spanning a large angular range, while maintaining the capability of transmitting and receiving, with the same performance metrics of a bare dipole antenna in the cellular band.

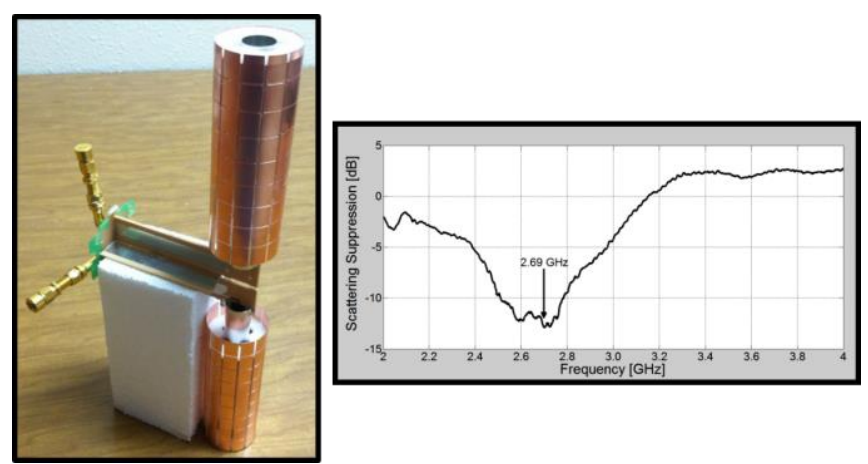

Figure 1: (Adapted from [18]-[19]) A cloaked dipole antenna, designed to transmit and receive radio-frequency signals, while being radio-transparent when excited by an arbitrary external field. On the right: measured scattering gain, defined as the ratio (in $d B$ ) between a bare dipole and a cloaked one.

\section{AN INVISIBLE ACOUSTIC SENSOR}

Cloaking a sensor or a receiving antenna is an interesting

Solid-State Sensors, Actuators and Microsystems Workshop Hilton Head Island, South Carolina, June 5-9, 2016 
application of metamaterials, not only for its impact on engineering applications, but also because it represents a fundamental challenge for basic science. The same action of sensing, in fact, requires extracting a portion of the impinging signal, and therefore it is expected to create a shadow [20]. A way around this fundamental limitation can be realized by considering active metamaterials, or artificial materials that involve active inclusions that can draw energy by a reservoir.

Inspired by recent advances in quantum mechanics in the area of parity-time (PT) symmetry, which studies systems whose Hamiltonian is symmetric under a concurrent parity and timereversal operation, we have recently shown that it is possible to realize a fully invisible acoustic sensor with strong absorption cross-section. The concept was based on pairing a resonant absorbing sensor with its time-reversed image (Fig. 2) [21]. In this device, whose loss and gain are balanced in space, an incoming signal, independent of its amplitude and phase, can be fully absorbed in the first element, yet at the same time the second element produces an exact replica of the incoming signal, eliminating shadows and reflections. The realized system was based on two identical loudspeakers loaded by circuit elements with conjugate impedances. The first loudspeaker was passive, and was designed to convert the impinging sound into a voltage across a resistor. At the same time, the second element was active, with the time reversed impedance of the first element, whose role was to emit a signal in sync with the impinging one, that would suppress the shadow created by absorption. Interestingly, the functionality of this pair of devices may be at the basis of a new class of metamaterials with PT-symmetric properties. We have envisioned fascinating applications of these metamaterials in acoustics and electromagnetics, including the possibility of realizing loss-free negative index, aberration-free planar lenses [22]-[23] and a new generation of advanced cloaking devices that go beyond the limitations of passive cloaks [24].
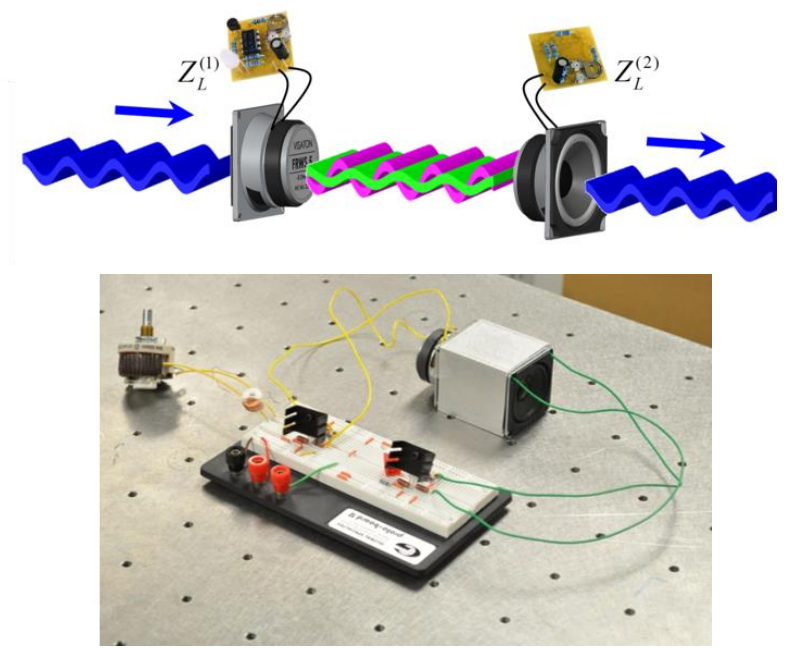

Figure 2: (Adapted from [19],[21]) An invisible acoustic sensor based on PT-symmetric loudspeakers achieved by loading them with conjugate impedances. This system enables a shadow-free efficient acoustic sensor that can pave the way to loss-free negative-index lenses and active cloaks that overcome the limitations of conventional cloaking technology.

\section{NON-RECIPROCAL MAGNETIC-FREE DEVICES}

Sound, radio-waves and light typically travel in space in a symmetric fashion: if we can send a signal from point $A$ to point $B$, we can expect to be able to send it back from B to A with equal strength. This inherent symmetry stems directly from Lorentz reciprocity theorem, and it is directly associated with the timereversal symmetry of wave propagation in conventional media. Reciprocity is not necessarily desirable, however, especially when the aim is to isolate a source from its echo, or separate signal flows traveling in opposite directions. An important application of nonreciprocal propagation is for full-duplex communications, i.e., the possibility of transmitting and receiving signals from the same antenna on the same frequency channel, leading to a more efficient use of the radio-wave communication spectrum, as well as better radar systems and ultrasound imaging devices.

The most common way to break reciprocity is based on breaking time-reversal symmetry by biasing ferromagnetic materials with a constant magnetic bias. This approach presents however several challenges: it requires the use of scarce materials, which also lead to heavy and pricy devices, and it is difficult to integrate them on-chip. Recently we have shown that reciprocity can be broken without the need of magnetic bias, replacing it with an angular-momentum bias properly applied to a metamaterial cavity. In our first proof-of-concept demonstration, we applied an angular momentum bias in the form of a slow motion of fluid, to a circularly symmetric acoustic cavity, enabling a first-of-its-kind circulator, or a three-port non-reciprocal device that imparts a preferred sense of circulation to the incoming signal, for acoustic waves (Fig. 3) [25].

The system is a basic three-port device that allows one-way rotation of the input signals, rotating from port 1 to 2 , from 2 to 3 , and from 3 to 1 , while it prevents transmission in the opposite circulation direction. Based on this simple system, we were able to measure very large isolation (over $40 \mathrm{~dB}$ ) for airborne acoustic waves. The subwavelength acoustic ring cavity shown in Fig. 3 was biased by moving air rotated simply using fans, and the cavity was symmetrically coupled to three acoustic waveguides, which formed the input and output channels of the device.

While it is interesting to see how such a basic component can dramatically modify the way in which sound propagates, not always a mechanical motion of the filling material may be convenient or practical, especially when translating these effects to other types of waves, such as radio signals, which travel much faster than sound. In [26]-[27] these concepts were extended to an equivalent meta-device in which fluid motion was replaced by a suitable form of spatio-temporal modulation of three strongly coupled resonators, which emulates an angular-momentum bias, enabling the realization of magnetic-free circulators for radio and ultrasound waves. We also extended these concepts to light, by considering a microring resonator biased by spatio-temporal modulation imparted via three $\mathrm{p}-\mathrm{i}-\mathrm{n}$ junctions. Using realistic parameters, we were able to design an integrated nanophotonic circulator for light tuned around telecom wavelengths [28]. 

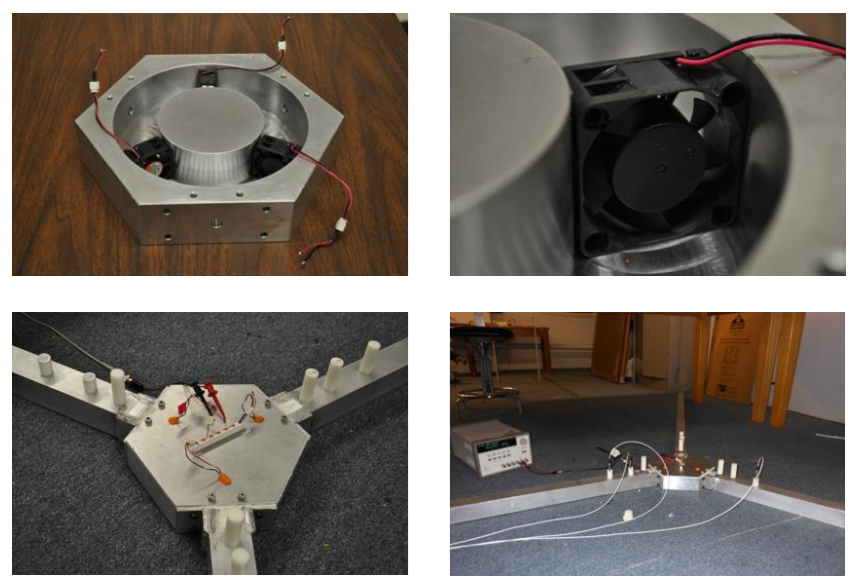

Figure 3: (Adapted from [25]) A first-of-its-kind circulator for acoustic waves, which enables large isolation and time-reversal symmetry breaking by applying an angular momentum bias to a circularly symmetric cavity. The cavity was loaded with three CPU fans that move air at a moderate velocity, fast enough to break reciprocity and enable large isolation. The cavity is connected to three acoustic waveguides carrying acoustic air-borne signals.

\section{CONCLUSIONS}

The field of metamaterials has opened exciting directions in basic research, with a direct impact on several applications, spanning electromagnetics, acoustics, nano-optics, and beyond. Metamaterials have become an important platform for applied technology, with implications at the frontiers of several engineering fields, opening exciting new directions and overcoming several of the conventional limitations of technology that stem from the use of conventional materials.

\section{ACKNOWLEDGMENTS}

This work was supported by the Air Force Office of Scientific Research, the Office of Naval Research, the Defense Threat Reduction Agency, and the National Science Foundation.

\section{REFERENCES}

[1] R. A. Shelby, D. R. Smith, and S. Schultz, "Experimental verification of a negative index of refraction," Science, 292, 77 (2001).

[2] J. B. Pendry, "Negative refraction makes a perfect lens," Phys. Rev. Lett. 85, 3966 (2000).

[3] N. Engheta, "An idea for thin subwavelength cavity resonators using metamaterials with negative permittivity and permeability," IEEE Antennas and Wireless Propagation Lett. 1, 10 (2002).

[4] A. Alù, and N. Engheta, "Guided Modes in a Waveguide Filled with a Pair of Single-Negative (SNG), DoubleNegative (DNG), and/or Double-Positive (DPS) Layers," IEEE Transactions on Microwave Theory and Techniques 52, 199 (2004).

[5] A. Alù, and N. Engheta, "Polarizabilities and Effective Parameters for Collections of Spherical Nano-Particles Formed by Pairs of Concentric Double-Negative, SingleNegative and/or Double-Positive Metamaterial Layers," Journal of Applied Physics 97, 094310 (2005).

[6] A. Alù, and N. Engheta, "Achieving Transparency with Plasmonic and Metamaterial Coatings," Physical Review E 72, 016623 (2005).
[7] J. B. Pendry, D. Schurig, D. R. Smith, "Controlling Electromagnetic Fields," Science 312, 1780 (2006).

[8] D. Schurig, J. J. Mock, B. J. Justice, S. A. Cummer, J. B. Pendry, A. F. Starr, and D. R. Smith, "Metamaterial Electromagnetic Cloak at Microwave Frequencies," Science 314, 977 (2006).

[9] R. W. Ziolkowski, and A. Erentok, "Metamaterial-Based Efficient Electrically Small Antennas," IEEE Transactions on Antennas and Propagation 54, 2113 (2006).

[10] A. Alù, "First-Principles Homogenization Theory for Periodic Metamaterials," Physical Review B 84, 075153 (2011).

[11] A. Alù, and N. Engheta, "Pairing an Epsilon-Negative Slab with a Mu-Negative Slab: Resonance, Tunneling and Transparency," IEEE Transactions on Antennas and Propagation, Special Issue on Metamaterials 51, 2558 (2003).

[12] D. R. Fredkin, and A. Ron, "Effective left-handed (negative index) composite material," Appl. Phys. Lett. 81, 1753 (2002).

[13] N. Engheta and R. W. Ziolkowski, Metamaterials: Physics and Engineering Explorations. John Wiley \& Sons, 2006.

[14] A. Alù, and N. Engheta, "Cloaking a Sensor," Physical Review Letters 102, 233901 (2009).

[15] F. Monticone and A. Alù, "Do Cloaked Objects Really Scatter Less?," Phys. Rev. X, vol. 3, no. 4, p. 041005, Oct. 2013.

[16] A. Alù, "Mantle Cloak: Invisibility Induced by a Surface," Physical Review B, Vol. 80, No. 24, 245115 (5 pages), December 21, 2009

[17] A. Monti, J. Soric, A. Alù, A. Toscano, L. Vegni, and F. Bilotti, "Overcoming Mutual Blockage Between Neighboring Dipole Antennas Using a Low-Profile Patterned Metasurface," IEEE Antennas and Wireless Propagation Letters, Vol. 11, pp. 1414-1417, December 20, 2012.

[18] J. Soric, A. Monti, A. Toscano, F. Bilotti, and A. Alù, "Antenna Blockage Reduction Using Metasurface Cloaks," IEEE Trans. Antennas Propagat. 63, 4027 (2015).

[19] A. Alù, "Metamaterial-Based Device Engineering," in 2015 U.S. Frontiers in Engineering, National Academy of Engineering, pp. 99-103, 2016

[20] R. Fleury, J. Soric, and A. Alù, "Physical Bounds on Absorption and Scattering for Cloaked Sensors," Physical Review B, Vol. 89, No. 4, 045122 (12 pages), January 15, 2014

[21] R. Fleury, D. L. Sounas, and A. Alù, "An Invisible Acoustic Sensor Based on Parity-Time Symmetry," Nature Communications, Vol. 6, No.5905 (7 pages), January 6, 2015.

[22] R. Fleury, D. Sounas, and A. Alù, "Negative Refraction and Planar Focusing Based on Parity-Time Symmetric Metasurfaces," Physical Review Letters, Vol. 113, No. 2, 023903 (5 pages), July 10, 2014.

[23] F. Monticone,* C. A. Valagiannopoulos,* and Andrea Alù, "Aberration-Free Imaging Based on Parity-Time Symmetric Nonlocal Metasurfaces," http://arxiv.org/abs/1509.07300.

[24] D. L. Sounas, R. Fleury, and A. Alù, "Unidirectional Cloaking Based on Metasurfaces with Balanced Loss and Gain," Physical Review Applied, Vol. 4, 014005 (11 pages), July 16, 2015.

[25] R. Fleury, D. L. Sounas, C. F. Sieck, M. R. Haberman, and A. Alù, "Sound Isolation and Giant Linear Nonreciprocity in a Compact Acoustic Circulator," Science, Vol. 343, No. 6170, pp. 516-519, January 31, 2014

[26] N. Estep*, D. Sounas*, J. Soric, and A. Alù, "Magnetic-Free Non-Reciprocity Based on Parametrically Modulated Coupled-Resonator Loops," Nature Physics, Vol. 10, No. 12, pp. 923-927, December 1, 2014. 
[27] R. Fleury, D.L. Sounas, and A. Alù, "Subwavelength Ultrasonic Circulator Based on Spatio-Temporal Modulation," Physical Review B, Vol. 91, No. 17, 174306 (9 pages), May 28, 2015.

[28] D. Sounas, and A. Alù, “Angular-Momentum-Biased Nanorings to Realize Magnetic-Free Integrated Optical Isolation," ACS Photonics, Vol. 1, No. 3, pp. 198-204, March 19, 2014.

\section{CONTACT}

A. Alù, tel: +1-512-471-5922; alu@mail.utexas.edu 